\title{
Intégration des Services au Sénégal
}

Population Council

Follow this and additional works at: https://knowledgecommons.popcouncil.org/departments_sbsr-rh

Part of the International Public Health Commons, Maternal and Child Health Commons, Public Health Education and Promotion Commons, Social and Behavioral Sciences Commons, and the Women's Health Commons How does access to this work benefit you? Let us know!

\section{Recommended Citation}

Population Council. 2010. "Intégration des Services au Sénégal," brief. Dakar: Population Council. 


\section{Sénégal}

Les services intégrés de Santé de la Reproduction (SR) permettent aux femmes d'accéder à de multiples services en général au même moment, dans le même lieu, et souvent par le même prestataire. Si les services sont bien intégrés, par exemple, une femme venue pour des services de consultation prénatale pourrait ainsi recevoir des services de conseils et dépistage des infections sexuellement transmissibles (IST y compris le VIH) et un traitement si nécessaire, mais aussi des conseils sur la planification familiale, la nutrition et l'hygiène. Des services intégrés permettent aux femmes d'accéder à une gamme plus large de services, d'informations et de soins au cours d'une même consultation et augmentent ainsi I'utilisation des services tout en améliorant la qualité et l'efficacité de ces services. ${ }^{1}$

\section{Modèles d'intégration testés dans Sénégal}

Avec le soutien financier de I'USAID et l'appui technique d'IntraHealth et de Population Council, du Réseau Siggil Jigeen et d'Helen Keller International, le Ministère de la Santé et de la Prévention du Sénégal a mis en place un paquet intégré de services de "Santé Maternelle, Néonatale et Infantile, Planning Familial, Lutte contre le Paludisme " (SMNI/PF/PALU).

Dans le cadre de la mise en œuvre de ce paquet intégré de services, trois modèles d'intégration spécifiques ont été expérimentés : 1) l'intégration des services de soins de la mère et du nouveau-né ; 2) l'intégration de la planification familiale dans tous les services de SR et ; 3) l'intégration de la prévention et de la prise en charge du paludisme dans les services de consultation prénatale, néonatale et postnatale.

L'intégration des soins maternels et néonatals est quelque chose de naturel et pratique dans la mesure où la mère et son bébé constituent un binôme où le bien-être de l'un dépend étroitement de l'autre. Ainsi, pour répondre simultanément aux besoins de santé des deux, un modèle intégré de services simple et peu coûteux est nécessaire. Au Sénégal, deux activités complémentaires -la Gestion Active de la Troisième Phase de l'Accouchement (GATPA) et les Soins Essentiels du Nouveau-né (SENN)-ont été combinées pour assurer un continuum de services pour la prise charge intégrée du couple mère-enfant. A cet effet, 689 prestataires de santé à travers sept régions ont été formés sur l'offre de ces services intégrés, dont la GATPA et les SENN. En conséquence, le nombre de services offerts a grimpé en flèche: 1600045 consultations prénatales, 470106 consultations postnatales, et 134904 accouchements sous GATPA ont été réalisées de Janvier 2008 à Décembre 2009.

Intégrer la planification familiale dans les autres services de SR permet de prendre en charge les besoins habituellement non satisfaits de la femme en matière de contraception au cours de son cycle reproducteur, notamment durant les périodes post-partum et post-avortement. Le paquet "Etre Mère" a été le second modèle d'intégration développé pour accroître les connaissances et les capacités des prestataires en matière d'offre de services de planification familiale dans le cadre d'un ensemble intégré de SR. Cette intégration des services de PF dans les autres services de SR a contribué à augmenter l'accès des femmes à l'information et aux services de PF : en 2009, 10.419 femmes ont bénéficié de services de soins post-avortement et $59 \%$ d'entre elles ont pu accepter une méthode de planification familiale après leur traitement.

Le paludisme a des effets particulièrement nocifs sur les femmes enceintes et leurs enfants, et par conséquent sa prise en charge devrait être possible aux endroits où ces clientes se rendent pour obtenir des soins, particulièrement dans les services de consultation prénatale, néonatale et postnatale. L'intégration de ces services a fortement augmenté l'accès à l'information, ainsi qu'aux services de prévention et de traitement du paludisme chez ces populations à risque. Ainsi, 357.867 femmes ont reçu un traitement préventif intermittent (TPI) et 408.983 femmes ont reçu une prescription de moustiquaire imprégnée d'insecticide durant la période prénatale, ce qui indique une bonne intégration des directives de prévention du paludisme lors de la consultation prénatale.

\section{Mise en œuvre de l'intégration}

Des interventions majeures initiées dans le cadre du projet SMNI / PF / PALU ont facilité la mise en œuvre de ces nouveaux modèles intégrés et permis de renforcer le système de santé sur lequel ils ont été construits. Deux initiatives spécifiques incluant une approche novatrice de formation du prestataire "Le Tutorat", et un système intégré d'information sanitaire ont contribué à consolider cette intégration des services.

"Le Tutorat" est une approche de formation sur le site basée sur les besoins réels de formation du prestataire, et qui
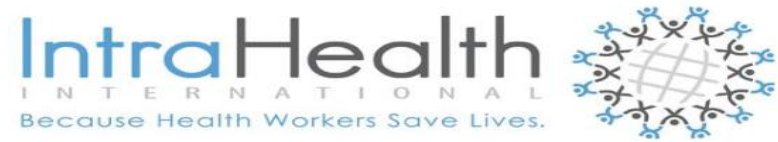

(2) Population Council 
favorise l'offre des services de santé intégrés de haute qualité avec une évaluation continue des performances des prestataires grâce à l'utilisation par le tuteur de fiches $d$ 'apprentissage/listes de vérification pour chaque élément du paquet SMNI/PF/PALU. Le tuteur est souvent un pair qui a acquis une bonne expérience pratique et qui a des compétences en matière d'encadrement. Après un recensement des besoins de l'agent, chaque prestataire reçoit une formation individualisée par un tuteur.

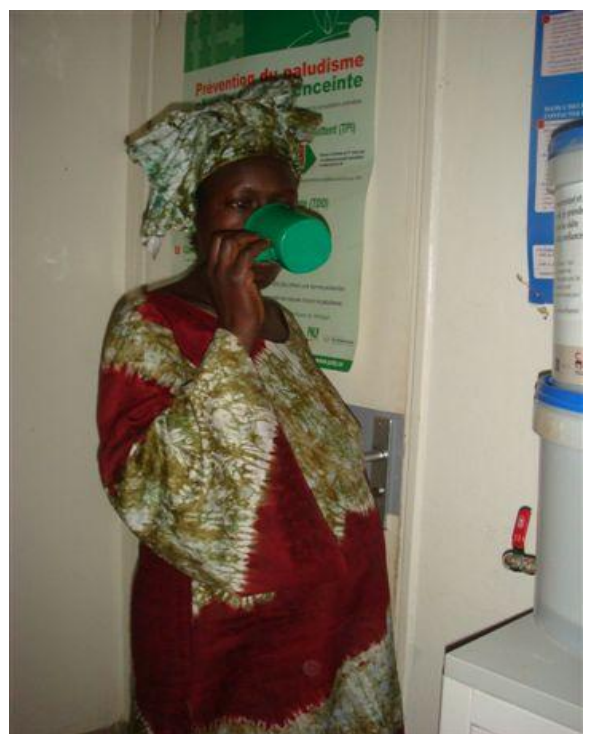

Une femme recevant le TPI lors d'une CPN

Contrairement aux approches de formation traditionnelles qui se concentrent sur un type de service ou un ensemble de compétences spécifiques, le tutorat met l'accent sur la fourniture de services intégrés et holistiques, notamment: les compétences en IEC et en counseling, la gestion logistique, et l'assurance qualité. II met surtout l'accent sur les facteurs de performance qui peuvent influencer sur la qualité des services (ex: l'équipement, l'organisation des services). De par son approche, le tutorat maintien le prestataire dans son lieu de travail et assure la continuité des services pour les clients des services. Une autre importante caractéristique du "Tutorat" a été l'incorporation de l'approche d'Identification Systématique des Besoins du Client (ISBC). L'ISBC est une stratégie permettant d'augmenter le nombre de services qu'une cliente reçoit lors d'une visite unique, et qui est basée sur l'utilisation d'une simple liste de contrôle permettant à un prestataire de poser des questions à la cliente et de fournir des services complets et intégrés.

Depuis la mise en œuvre du tutorat, 573 prestataires ont été formés [dont 213 prestataires qualifiés incluant des sagesfemmes (137), des infirmiers/assistants infirmiers/agents sanitaires (76) et 360 travailleurs communautaires]. L'offre de services intégrés, l'environnement de travail, et l'organisation des services se sont considérablement améliorés.

Un système d'information sanitaire solide est également nécessaire pour asseoir l'offre de services intégrés. Au Sénégal, la Division de la Santé de la Reproduction (DSR) recueille ses propres données de routine sur les services de SR offerts au niveau des Points de prestations de services et la liste et les types de données devant être recueillies et consolidées à l'échelle nationale. Ainsi, avec le soutien du projet SMNI / PF /PALU, le SNIS et la DSR se sont réunis et ont procédé à l'harmonisation des données à collecter et des indicateurs de performance du programme SR.

De manière consensuelle, neuf indicateurs clés ont été sélectionnés pour suivre la qualité et l'offre de services de santé maternelle et néonatale. Les formulaires de collecte de données ont été révisés pour faciliter la collecte d'informations pertinentes sur la qualité et l'offre de nouveaux services intégrés, tels que le GATPA et les SENN. Les prestataires ont été formés sur le remplissage de ces nouveaux outils de gestion de l'information sanitaire. Cette collaboration a contribué à asseoir un système de collecte de données plus fiable qui est actuellement été étendu aux 14 régions du Sénégal.

\section{Conclusion}

L'intégration des services est un élément central du succès du projet SMNI / PF / PALU. En conjonction avec les modèles intégrés de prestation de services, les activités qui renforcent les systèmes de santé et qui appuient la mise en œuvre efficace des services demeurent pertinentes pour une intégration réussie. Ces actions ont démontré des effets positifs significatifs sur la santé des femmes et des enfants au Sénégal. Ainsi, une sage-femme travaillant dans un poste de santé dans la région de Thiès, Madame Sow Céline Nataye, déclare: «Avant l'intégration, nous avons connu beaucoup de problèmes avec les hémorragies post-partum et le la prise en charge des nouveau-nés. Mais maintenant, après la formation, les prestataires savent comment gérer ces complications ainsi que les gestes nécessaires pour sauver les vies des mères et des nouveau-nés. Maintenant, les prestataires formés sont capables de faire plus."

Juin, 2010

${ }^{1}$ Askew, 2009; Church and Mayhew 2009; Hardee et al 2009; WHO et al 2009
Ce résumé a été rendu possible grâce à l'appui apporté par le peuple américain à travers l'Agence Américaine Pour le Développement International

(USAID).Les contenus sont de la seule responsabilité des auteurs et ne reflètent pas nécessairement les vues de l'USAID ou du gouvernement des Etas Unis 